\title{
Natural Material Short Fibre Composites via Additive Manufacturing
}

\author{
Patrick Fairclough $^{\mathbf{1}}$, Stella Manoli ${ }^{1}$, Chris Holland ${ }^{2}$ \\ ${ }^{1}$ Department of Mechanical Engineering, The University of Sheffield \\ Sheffield, S1 4BJ, UK \\ P.fairclough@sheffeild.ac.uk \\ ${ }^{2}$ The Department of Materials Science and Engineering, The University of Sheffield \\ Sheffield, S1 3JD, UK
}

The influence of fibre orientation on the mechanical properties, of short fibre composites created by fused deposition modelling (FDM), was determined. Additionally, natural fibres of nettle and flax, with either minimal processing or with standard commercial processing were studied to determine how processing degrades the mechanical properties. The matrix material was a modified PLA, Floreon ${ }^{\circledR}$, that shows enhanced stiffness and toughness. This material was chosen to be chemically compatible with the natural fibres and to facilitate related studies into to the degradation profile. Test pieces were prepared with fibre orientation at $\pm 45^{\circ}$ and $0,90^{\circ}$ and filling fractions from $40-100 \%$. All samples showed improved mechanical properties over injection moulded samples.

Polylactic Acid is commonly used in 3D printing applications and FDM, in particular, is a common processing technique. The major issue with FDM is that the parts tend to show poor toughness, porosity and warping on cooling may be an issue. Floreon ${ }^{\circledR}$ was chosen partly due to historic reasons, it was codeveloped at the University of Sheffield, and due to its chemical compatibility with cellulose based fibres. Local flax and nettle were harvested, dried, cut into short fibres by minimal processing, specifically designed to maintain the fibre integrity and therefore the strength and stiffness. These were extrusion compounded with Floreon®, into the FDM filament.

A Lazbot TAZ 3 FDM system was used, as this is a highly customisable system that allows access to all the processing parameters. A wide range of processing parameters were studied, in an attempt, to deliver an optimal print output. In particular, the fibrous nature of the filament required careful monitoring of the FDM extrusion rate, temperature and layer thickness.

Results will be presented showing the tensile and flexural properties of the FDM versus injection moulded samples. The results will be discussed with consideration for the orientation of both the fibres and the polymer matrix. The relationships between the FDM processing parameters and the observed properties will also be presented. 